\title{
I DIRITTI UMANI E LA SFIDA DELL’UNIVERSALITÀ
}

\author{
HUMAN RIGHTS AND THE CHALLENGE OF UNIVERSALITY
}

Alessandra Algostino ${ }^{1}$

\footnotetext{
Recebido em: 26/07/2016 Aceito em: 15/09/2016

alessandra.algostino@unito.it
}

Summario: Ancora prima della Loro Nascita i Diritti Umani rivelano la Loro Ambiguità; quando si Tratta di scansione e in concreto la Loro Astratta Validità per Qualsiasi Umana persona, si inseriscono delle graduazioni nell'umanità della persona Che consentono giustificare di un Trattamento differenziato: per Vitoria, è stato Stato di Minoriti, Oggi è la cittadinanza?

Abstract: Even before the Loro Birth Human Rights reveal Their ambiguity ; When it Comes to scan and concretely Their Abstract Any validity to the individual Human, are part of the graduations in the humanity of the person allow That justify a differentiated treatment: Vitoria , was the State of Minoriti, today is your citizenship?

\section{INTRODUZIONE}

\section{Premessa $^{2}$}

L'idea di diritti umani universali, dote di ciascuna persona umana, possiede un fascino irresistibile e non si contano le teorie che ne hanno argomentato il fondamento, descritto la nascita e l'evoluzione, illustrato il catalogo: è una chimera?

Proveremo a rispondere ascoltando il canto della sirena saldamente legati ad un ruvido albero maestro, navigando dapprima nell'empireo delle teorie dei diritti umani e poi, scendendo sulla terraferma, accostandoci alle ambiguità della loro concretizzazione.

In un arcipelago costellato di isole, si sceglie di accostarsi solo ad alcune, senza dunque alcuna pretesa di una (impossibile) completezza e senza pretendere di scegliere l'optimum, ma tentando semplicemente di seguire un fil rouge che aiuti a disvelare i retroscena dell'attrazione fatale dell'universalità.

Come carta nautica per la navigazione si distinguono le teorie fra quelle che trattano dell'universale: a) sotto il profilo del fondamento; b) creando una procedura per individuarne il contenuto; c) muovendosi sul piano della definizione formale.

\section{ALLA RICERCA DI UN FONDAMENTO UNIVERSALE: DAL GIUSNATURALISMO AL POSITIVISMO, ATTRAVERSANDO LE TEORIE STORICHE E L'APPROCCIO RAZIONALISTA}

Muovendo dalle teorie sul fondamento, si possono citare due metagruppi, tradizionalmente considerati agli antipodi: giusnaturalismo e positivismo.

È il giusnaturalismo ad introdurre, nella storia dei diritti, l'idea di titolarità universale in quanto discendente da una comune natura umana: «madre del diritto naturale è la stessa natura umana»

\footnotetext{
${ }^{1}$ Università di Torino - UNITO - Itália.

2 Per riferimenti bibliografici, e per un approfondimento dei temi trattati, ci si permette di rinviare a A. Algostino, L'ambigua universalità dei diritti. Diritti occidentali o diritti della persona umana?, Jovene, Napoli, 2005.
} 
(GROZIO, 1948). É un diritto i cui principi sono «per sé chiari, ed evidenti», legati alla facoltà raziocinante dell'uomo ed espressione di «una conseguenza procedente da principi di natura» (GROZIO, 1948, p. 73/74). Permangono a lungo, peraltro, rapporti ambigui con la religione. Per una indifferenza nei confronti del fattore religioso, occorre passare per l'llluminismo, le guerre di religione, e giungere sino alla fine del Settecento; senza scordare, tuttavia, che sia in Locke sia nella Dichiarazione dei diritti della Virginia del 1776 la libertà di religione si accompagna ancora ad un rifiuto dell'ateismo ${ }^{3}$. La religione non è comunque l'unico elemento che inficia l'universalità della comune natura umana, anzi, è interessante notare come sin dalle prime teorizzazioni del diritto naturale la sua proclamata universalità ceda alla realpolitik. Emblematico in tal senso è il pensiero di Vitoria.

II dominium - argomenta Vitoria - spetta all'essere umano in virtù della sua condizione naturale, a prescindere dall'eventuale stato di peccatore e/o dall'osservanza della religione (cristiana): compete alle creature razionali. Dunque, trovandosi di fronte i "selvaggi" abitanti delle Indie, peccatori e "infedeli", Vitoria sostiene che possono essere titolari del dominium, a condizione ovviamente che siano razionali. Ed in quest'ultima precisazione sta l'escamotage: i barbari possono essere riconosciuti tendenzialmente razionali e, quindi, capaci di dominium, ma nello stesso tempo sono come dei minori, bisognosi di essere guidati (e sottomessi) agli uomini pienamente razionali ${ }^{4}$.

La potenziale universalità del diritto naturale si piega alle esigenze della colonizzazione. Gli indios, "veri domini» in astratto, divengono in concreto «hebetes», e i diritti, appartenenti a tutti gli esseri razionali, sono asimmetrici e strumentali rispetto agli interessi degli Spagnoli, come è evidente se si scorre il loro elenco: ius peregrinandi et degendi, ius commercii, ius occupationis, ius migrandi, ius praedicandi et annuntiandi Evangelium ${ }^{5}$.

Ancora prima della loro nascita i diritti umani rivelano la loro ambiguità; quando si tratta di verificare in concreto la loro astratta validità per qualsiasi persona umana, si inseriscono delle graduazioni nell'umanità della persona che consentono di giustificare un trattamento differenziato: per Vitoria, era lo stato di minorità, oggi è la cittadinanza?

Se si passa alla prime traduzioni del diritto naturale in diritto positivo, ovvero le dichiarazioni di fine Settecento, che possono assumersi quali atti di nascita dei diritti dell'uomo, a revocare in dubbio il riconoscimento dei diritti in capo a tutti appare proprio la cittadinanza. A tacer del fatto che, concretamente, il termine «uomo» sottintende il rinvio ad una persona umana di sesso maschile, possidente e bianca, la Dichiarazione francese dei diritti dell'uomo e del cittadino del 1789 oscilla nell'attribuzione dei diritti fra uomo e cittadino. Oltre che nel titolo stesso, ciò emerge con evidenza

\footnotetext{
${ }^{3} \mathrm{Cfr}$. Locke, che riconosce la libertà religiosa, in quanto fatto meramente individuale, e, tuttavia, assume una posizione intollerante nei confronti dell'ateismo: «non devono essere assolutamente tollerati quelli che negano che ci sia una divinità.... (J. Locke, Epistola de Tolerantia, 1689, trad. it. Lettera sulla tolleranza, Laterza, RomaBari, 1998, p. 45); quanto alla Dichiarazione dei diritti della Virginia, si può ricordare come, nel sancire la libertà religiosa (sez. 16), si riferisca al «dovere che abbiamo verso il nostro Creatore».

${ }^{4}$ F. De Vitoria, Relectio de Indis. La questione degli Indios (1539), edizione latino-italiano, curata da A. Lamacchia e ripresa dalla edicion critica bilingue por L. Pereña y J. M. Perez Prendes (Relectio de Indis o libertad de los Indios, Consejo superior de investigaciones cientificas, Madrid, 1967), Levante Editori, Bari, 1996: gli indios «non sunt amentes, sed habent pro suo modo usum rationis...» (Prima pars, Sectio I, 15, p. 29), ma, nel caso essi siano "inepti et hebetes», "ex hac ratione et titulo posset oriri aliquod ius ad subiciendum eos» (Prima pars, Sectio I, 16, p. 31).

${ }^{5}$ Cfr. F. Vitoria, Relectio de Indis, cit., Prima pars, Sectio III, pp. 78 ss.
} 
nell'art. 11, laddove recita «la libera comunicazione dei pensieri e delle opinioni è uno dei diritti più preziosi dell'uomo» e «ogni cittadino può dunque parlare, scrivere e stampare liberamente, ...» ${ }^{6}$.

Oltreoceano, invece, la proclamazione dell'eguaglianza di tutti gli uomini convive con il «we» della Dichiarazione di indipendenza del 4 luglio 1776, che differenzia "cittadini americani" ed "altri", o con la presenza legale della schiavitù.

Entrambe le dichiarazioni, poi, incontrano, i limiti intrinseci alla positivizzazione, ovvero i confini territoriali del soggetto dal quale proviene il riconoscimento normativo: appare un altro potente fattore contro l'universalità, la sovranità degli Stati.

L'idea, comunque, di diritti ancorati all'uomo stesso, come essenza universale, permanente e data, esercita la sua attrazione ancor oggi, anche se il giusnaturalismo moderno è tendenzialmente meno astratto e tende a presentarsi più come espressione di un ragionamento logico che come oggetto di una ipotesi filosofica. Molte fra le moderne teorie giusnaturalistiche costruiscono in maniera discorsiva la natura umana e/o la concepiscono come insieme di possibilità, sottolineando elementi quali la scelta, la volontà, il consenso, l'auto costruzione e determinazione, e trascurando l'immagine della natura umana quale componente di un ordine cosmologico. Resta l'idea di un quid universale che sia l'uomo lockiano con i suoi diritti naturali o una natura umana concepita come «telos, compimento, pieno sviluppo» (BERTI, 1987, p. 290) che fonda la titolarità (di diritti) in capo a tutti.

Il positivismo delle costituzioni moderne o delle dichiarazioni continentali e internazionali dei diritti umani, se pur con i limiti legati all'ambito territoriale di validità delle norme, si pone con lo stesso afflato universalista del diritto naturale. Ora, non è tanto la considerazione delle costituzioni come «processo di positivizzazione del diritto naturale», il loro essere «tavole positive di diritto naturale» (FERRAJOLI, 1989), ma l'operare del positivismo come il diritto naturale, con le sue caratteristiche di "intoccabilità" e universalità. Ciò che si ripresenta nel diritto costituzionale moderno o nel diritto internazionale dei diritti umani è la «funzione storica costante del giusnaturalismo»: rispondere all'«eterna esigenza... che la vita, alcuni beni e alcune libertà dell'individuo siano protette giuridicamente contro la forza organizzata di coloro che detengono il potere», sottrarre la natura umana «alla mutevolezza della storia, fondarla oggettivamente, attribuirle un valore universale...» (BOBBIO, 1962, p. 67-79). L'elemento connotante è la volontà di sancire una prospettiva "protetta", che sia non solo descrittiva ma anche prescrittiva. È un intento che è di immediata evidenza nel giusnaturalismo, più appannato nel positivismo, che, con le costituzioni o il diritto internazionale consuetudinario, coniuga il desiderio di "eternità" con la storicità di un diritto, e di diritti, frutto di conflitti contestualizzati in un dato habitat sociale, economico, politico e culturale (AZZARITI, 2010).

È una tensione, quella verso la permanenza, che, nel suo tutelare i diritti di ciascuna persona umana, trascina con sé anche la loro connaturata universalità, e attraversa non solo il giusnaturalismo e il positivismo, ma anche le teorie cosiddette tradizionaliste e/o storiche, che saldano il riconoscimento dei diritti al trascorrere del tempo, o l'approccio c.d. razionalista, che ancora i diritti ad alcuni valori (quali giustizia e democrazia), o alla "fiducia" (fede?) in una determinata procedura. Se si vuole individuare una differenza rispetto al giusnaturalismo (o, analogamente, rispetto alle ipotesi che fondano i diritti in una sfera metafisica, religiosa o non), la si può individuare nell'osservazione che la

\footnotetext{
${ }^{6}$ Si veda anche il passaggio «diritti naturali», «legge», «cittadini» negli articoli 4, 5 e 6.
} 
"stabilità" dei diritti nelle altre scuole di pensiero ricordate è argomentata come un fatto empirico e volontaristico più che assunta come un oggetto di fede.

Si pensi alle teorie storiche: i diritti sono creati dagli uomini anche se il decorrere del tempo li sublima, conferendo loro un'aura di inviolabilità, per cui assurgono al rango di «inalienabile eredità» (BURKE, 1963, p. 192). Non solo, la storia mostra come anche l'evoluzione nel senso della titolarità universale è una conquista che avviene progressivamente con il trascorrere dei decenni e dei secoli. L'esperienza inglese è emblematica: i diritti della Magna Charta Libertatum, nati nel 1215 come privilegi delle classi sociali che avevano avuto la forza di strapparli al sovrano, divengono i diritti degli inglesi ${ }^{7}$, per poi migrare nel catalogo dei diritti dell'uomo. Certo, la sedimentazione non esclude virate "all'indietro", e non solo sul piano dell'effettività, ovvero della violazione e della concreta garanzia dei diritti, ma anche su quello della proclamazione ${ }^{8}$. Si pensi alla restrizione della libertà personale che accompagna gli stati di emergenza o, più "semplicemente", l'esigenza di controllare le frontiere, o la progressiva dismissione della tutela pubblica del diritto allo studio o alla salute. Spesso, fra l'altro, l'inversione avviene anche nella sfera della titolarità, diversificando ad esempio il trattamento dei cittadini da quello degli stranieri.

Quanto all'approccio razionalista, esso costruisce i diritti come razionale e logica implicazione deducibile (o, meglio, a volte, "inducibile") da un valore di partenza, sostanziale o procedurale: i diritti sono quei "precetti" che gli uomini individuano come intimamente connessi ad un certo scopo, principio, o modus procedendi ${ }^{9}$. L'orizzonte è dunque di tipo artificiale e volontarista, costruttivista; spesso si utilizza un metodo di tipo razionale e si insiste sull'accordo e sul consenso, anche se non mancano scivolamenti verso presupposti assunti come dati, quali la razionalità 0 il velo dell'ignoranza $^{10}$. Sono tesi spesso caratterizzate ab origine da un approccio universalista; centrale è l'individuazione del quid universale, muovendo dal soggetto "uomo", con la sua razionalità ma anche con le sue differenze. Si naviga nei mari in cui, lasciata la terra dell'uomo con la sua dote di diritti

\footnotetext{
${ }^{7}$ Si veda l'insistenza di E. Burke, Riflessioni sulla Rivoluzione francese, cit., p. 192, nel considerare i diritti «proprietà appartenente in modo speciale al popolo di questo regno».

${ }^{8}$ Non è questa la sede, ma si ricorda il dibattito tra "ottimisti" e "pessimisti" quanto alle tendenze umane: $i$ diritti nascono da una naturale propensione dell'uomo al "bene" o dalla volontà di arginare un "homo homini lupus"?

9 Si vedano, per l'argomentazione dei diritti come "discesa" da un valore, ad esempio, J. Rawls, La legge dei popoli, in S. Shute, S. Hurley (a cura di), I diritti umani. Oxford Amnesty Lectures 1993, Garzanti, Milano, 1994, p. 82: «i diritti umani fondamentali sono espressione di uno standard minimo di istituzioni politiche bene ordinate per tutti i popoli che appartengono, in qualità di membri a buon diritto, a una giusta società politica dei popoli»; R. A. Dahl, On Democracy, 1998, trad. it. Sulla democrazia, Laterza, Roma-Bari, 2000, pp. 52-53: «i diritti sono indispensabili alle istituzioni politiche democratiche, ...essi sono tra $\mathrm{i}$ fondamentali materiali costitutivi di una forma di governo democratica»; o, M. Walzer, Due specie di universalismo, in MicroMega, 1991/1, pp. 142-143, per il quale i diritti potranno (come si vedrà il suo universalismo è "debole") essere la risposta alla volontà di liberarsi dall'oppressione, ovvero dall' «esperienza dell'umiliazione o degradazione»; mentre, per le tesi che insistono in specie su una procedura, si rinvia al paragrafo infra.

${ }^{10}$ II riferimento è in specie a J. Rawls, A theory of justice (1971), trad. it. Una teoria della giustizia, Feltrinelli, Milano, 1997, pp. 125 ss., il quale presuppone che gli agenti siano «razionali» (o, meglio, ugualmente razionali) e, dunque, capaci di individuare le ragioni «appropriate», secondo una procedura "ragionevole», e ritiene che tale razionalità possa al meglio esplicarsi nella "posizione originaria», "sotto il velo dell'ignoranza»; si può peraltro notare come negli scritti più recenti (quali Political Liberalism, 1993, trad. it. Liberalismo politico, Edizioni di Comunità, Torino, 1999), Rawls tende a sottolineare maggiormente il significato procedurale dei principi di giustizia.
} 
naturali, si cerca la rotta, attraverso il pluralismo, per l'universale. È un universale da individuare e costruire.

\section{LA COSTRUZIONE DEL QUID UNIVERSALE TRA RELATIVISMO E PLURALISMO}

Si muove in queste ipotesi dall'osservazione delle differenze, in primo luogo culturali, per verificare l'esistenza di un eventuale nucleo comune a tutte le persone e disegnarne i confini ed i contenuti. Elemento cardine diviene l'individuazione di una procedura: la sfida è la via per una "autentica" universalità. Alcuni, poi, insistono sulla ricerca di un cammino gnoseologico, per trovare le coordinate universali; altri, costruiscono la strada per creare l'universalità. La prospettiva rispetto all'esistenza dell'universalità è, per così dire, ottimista: si confida di raggiungerla, senza lasciarsi sedurre dal primo incontro, accedendo ad un relativismo rinunciatario.

Emblematica in tal senso è l'interpretazione di un noto aneddoto raccontato da Erodoto: «una volta Dario, quando era re, convocò i Greci che venivano alla sua corte e domandò loro a quale prezzo avrebbero acconsentito a mangiare i cadaveri dei loro genitori; quelli dichiararono che per nulla al mondo l'avrebbero potuto fare. Allora Dario fece venire davanti a sé, presenti i Greci, quegli Egiziani, che sono chiamati Callati e che usano divorare i genitori morti e fece loro chiedere a mezzo degli interpreti per quale prezzo si sarebbero indotti a bruciare il cadavere del loro padre; e quelli a gran voce lo pregarono di non dire cose cosi sacrileghe ...» (D'AGOSTINO, 1996). Erodoto, dall'aneddoto, trae una conclusione relativistica, ma si può osservare come vi sia in realtà, pur se espresso con modalità all'apparenza inconciliabili, un valore fondamentale comune (il rispetto per i genitori). La relatività delle culture non implica l'impossibilità di ipotizzare un quid universale: le culture non sarebbero, dunque, votate ad uno scontro (HUNTINGTON 2000). ed i diritti umani non sarebbero una «insensatezza».

A sostenere la possibilità di individuare, attraverso un metodo essenzialmente induttivo, basato sull'osservazione nel tempo e nello spazio, delle costanti, le quali emergono come valori o finalità ultime rispetto a prassi, comportamenti o regole anche differenti fra loro, è, ad esempio, Walzer: non vi sono principi dati, ma «l'esperienza della reiterazione» consente di cogliere «la diversità delle rivendicazioni» e, nel contempo, che «le rivendicazioni fatte da noi le fanno anche altri» (WALZER, 1991, p. 141). È un «universalismo reiterativo», minimale e "sottile" (thin): si ragiona di «comunanze differenziate», di «pluralità di insiemi, parzialmente coincidenti, ognuno dei quali ha in comune con gli altri certi lineamenti», alcuni «tratti comuni... incorporati entro un particolare sistema culturale e elaborati in modi altamente specifici» (WALZER, 1991, p. 139/140). È un universalismo caratterizzato da un «punto focale particolaristico» e da una «tendenza pluralizzante» (WALZER, 1991, p. 131), molto "vuoto" e "insicuro": la sostanza dei valori comuni è di ardua individuazione, poco consistente e piuttosto instabile ${ }^{11}$.

\footnotetext{
${ }^{11}$ Più ricca appare invece l'«identità comune di base» se, dal livello mondiale, si scende a quello della singola società (cfr. M. Walzer, Sulla tolleranza, cit., spec. pp. 106-107); è lo stesso discorso che si può fare per il «patriottismo costituzionale» di J. Habermas (spec. Lotta di riconoscimento nello stato democratico di diritto, in J. Habermas, C. Taylor, Multiculturalismo. Lotte per il riconoscimento, Feltrinelli, Milano, 1998, p. 94, ma
} 
Veca, invece, identifica i diritti umani «nel centro focale dell'area del consenso per intersezione», quali «diritti umani politicamente neutrali» (VECA, 1997, p. 373). Si assume la presenza di un contesto plurale e, «in una situazione di simmetria fra i commentatori che sono sotto velo di ignoranza quanto alle concezioni del bene di ciascun partecipante», si scambiano i «commenti sulle circostanze in cui una vita umana non è degna di essere vissuta»: «l'insieme dei commenti che precede il riconoscimento della differenza fra i commentatori è...l'area di consenso per intersezione nel cui centro focale vi è la tesi sui diritti umani» (VECA, 1997, p. 375). L'universalismo è l'esito di «una procedura costruttiva» che si può definire dinamica: i diritti umani si fondano «sull'edificio in permanente costruzione e ricostruzione dei commenti su che cosa si prova a vivere vite umane» (VECA, 1997, p. 241 e 374). Peraltro, da un lato, si può rilevare, nonostante l'approccio costruttivista, la presenza di assunti presupposti, come l'“esistenza” del velo di ignoranza. Dall'altro lato, si può osservare come il pluralismo tenda a scomparire, anche se rimane sotto il profilo della legittimazione (sono i contesti, le storie diverse, che possono fornire le proprie ragioni per proteggere i diritti umani), nel momento in cui si individua l'universalità: essa, infatti, è costituita da «quanto i commentatori possano continuare a dire... sino a quando non cominci a divenire chiara e riconoscibile la differente identità dei commentatori» (VECA, 1997, p. 375). In sintesi, Veca sembra porre l'accento, più che sulla ricerca del minimo comun denominatore fra le culture, sull'individuazione degli elementi comuni oltre le culture, pur riconoscendone il valore plurale. È un universale comune non solo scarno (VECA, 2003, p. 117) ma anche "in negativo", nel senso che la convergenza è in specie su ciò che è male: «l'idea del bene ci divide, mentre ciò che può unirci è l'idea del male» (VECA, 2003, p. 115). I diritti umani si situano al confine, a perimetrare la regione del male, quella «in cui redigiamo l'indice dei mali sociali primari», prima delle «interpretazioni distinte e variegate» della regione del bene: solo in tal modo è possibile «sostenere, al tempo stesso, le ragioni dell'universalismo dei diritti umani e le ragioni del pluralismo morale, religioso e culturale» (VECA, 2003, p. 121/122).

Molto chiaro sul punto è Ignatieff (2003, p. 58): «chi proviene da culture diverse può continuare a essere in disaccordo riguardo a ciò che è il bene, ma nondimeno essere d'accordo su ciò che è insopportabilmente e indiscutibilmente sbagliato. Gli impegni universali implicati dai diritti umani possono essere compatibili con un'ampia varietà di modi di vivere solo se l'universalismo sotteso è consapevolmente minimalista. I diritti umani possono meritare l'assenso universale solo in quanto teoria decisamente "leggera" di cos'è giusto, in quanto mera definizione delle condizioni minime per ogni genere di vita». In altri termini, «il potenziale universalizzante» dei diritti umani deriva dalla «loro portata negativa (ciò contro cui insorgono)» che «è infinitamente più ampia della loro estensione positiva (ciò a cui aderiscono)» (JULLIEN, 2010, p. 124-125).

La condivisione in negativo attrae: ma è veramente così diversa? Ciò che è «insopportabilmente e indiscutibilmente sbagliato» (Ignatieff), o i «mali sociali primari» (Veca), non sono il risvolto di ciò che per tutti è il bene minimo, essenziale? Entrambi gli Autori nel riferirsi al «male» sembrano enuclearne una nozione minima: non si può individuare tutto ciò che è male per tutti, ma, semplicemente, ciò che per tutti è male, il "sommo" male (il quid comune del male). Se è

anche Id., La costellazione postnazionale. Mercato globale, nazioni e democrazia, Feltrinelli, Milano, 1999, p. 95).

Revista do Direito [ISSN 1982-9957]. Santa Cruz do Sul, v. 2, n. 49, p. 4-21, maio/ago. 2016. https://online.unisc.br/seer/index.php/direito/index 
vero che il contrario di ciò che è male per tutti non è necessariamente ciò che è il Bene per tutti, è anche vero che è bene per tutti: la prospettiva minimalista negativa si avvicina a quella positiva.

In sintesi, pare di poter rilevare come, anche senza accedere immediatamente ad un relativismo che nega la possibilità di costruire un universale, le procedure per una universalità che si ponga come condivisa e non presupposta, o imposta, mostrano la difficoltà di immaginare un universale prescindendo dalle culture. Latouche (2004, p. 19) osserva come «non esistono valori che siano trascendenti rispetto alla pluralità delle culture», perché «un valore esiste come tale solo in un contesto culturale dato», per cui occorre sostituire il sogno universalista, "con un «pluriversalismo» necessariamente relativo, cioè una vera «democrazia delle culture»» ${ }^{12}$. Questo non significa necessariamente rinunciare all'universale; come nota Sen (2004, p. 67): «non conosco alcun valore... contro il quale non siano state sollevate obiezioni. A mio parere, non è questo ciò che conta per considerare qualcosa come un valore universale. Al contrario, l'essenziale è stabilire se in ogni parte del mondo gli uomini possano avere ragioni per considerarlo tale».

Può aiutare in questo compito l'antropologia. Geertz (1999, p. 19), ad esempio, data la molteplicità delle culture, ritiene che «gli appigli per navigare in un mondo frammentato verranno alla luce solo grazie a un paziente e modesto lavoro di avvicinamento». Rouland (1998, p. 260) sottolinea come il canto dei diritti umani sia «polifonico», come l'idea stessa di dichiarazione universale dei diritti dell'uomo «può essere recepita, nelle diverse aree del mondo, solo in maniera differenziata» e, ancor prima, come una "concretizzazione storica» dei diritti è possibile solo se la loro formulazione risulta «da interpretazioni incrociate delle concezioni operanti nei diversi contesti culturali».

Per altri, poi, il primo ostacolo è già il termine "diritti" per cui si suggerisce di trovare l'«equivalente omeomorfo» del concetto di diritti umani, ovvero il quid che in un altro contesto culturale esercita la stessa funzione assolta in ambito occidentale dai diritti umani ${ }^{13}$; questo può condurre, ad esempio, ad ipotizzare una equivalenza tra la nozione di diritti umani e quella indiana di «svadharma» (il dharma che riguarda tutti gli esseri). $\mathrm{O}$, ancora, si propone, sempre nell'ambito di un approccio transculturale, il linguaggio delle "capacità», complementare rispetto a quello dei diritti umani, ma considerato meno ambiguo (NUSSBAUM, 2002). Le capacità umane fondamentali rappresentano in sostanza gli «elementi necessari a un funzionamento autenticamente umano», costituiscono delle «pretese della possibilità di funzionare» (NUSSBAUM, 2002, p. 74/80) e sono individuabili attraverso un discorso transculturale, rimanendo aperte sia ad una riscrittura sia ad una contestualizzazione.

II relativismo non conduce necessariamente al nulla, ma può significare molteplice coesistenza di valori; un relativismo aperto e "ottimista" può convivere con un universalismo senza mire assolutistiche e parco. È un universalismo pluriculturale e con-costruito, che piace definire situato. Si immagina, quindi, una universalità come frutto di una condivisione, a partire dall'esistenza

\footnotetext{
${ }^{12}$ per inciso: la "democrazia delle culture» non viene ad essere un valore universale ("deve" esser riconosciuto da tutti), se pur magari di tipo procedurale?

${ }^{13}$ Cfr. R. Panikkar, Ė universale il concetto di diritti dell'uomo?, in Volontari e Terzo Mondo, 1990/12, pp. 24 ss., nonché Id., La torre di Babele. Pace e pluralismo, Edizioni Cultura della Pace, San Domenico di Fiesole (FI), 1990, spec. pp. 48-50, ricordando che egli muove dalla considerazione che la cultura moderna nel «suo slancio di infinito», nella "sua sete di assoluto che la spinge a diventare universale», si autodistrugge: "ogni pretesa di assoluto scoppia, più o meno violentemente, nel regno del relativo» (op. ult. cit., pp. 144 ss.).
} 
di una pluralità di storie e culture ${ }^{14}$, in una prospettiva che si può qualificare interculturale ${ }^{15}$. È una universalità non fideisticamente ancorata a concetti presupposti, quale la Natura, o dedotta attraverso implicazioni logiche, ma legata alla storia e alla concreta condizione umana, dunque a persone uguali, ma anche diverse, "contestualizzate". L'universalismo è possibile, in quanto se ne accetti (perlomeno rebus sic stantibus) una versione "debole", basata su un nucleo, per così dire, molto ristretto, essenziale, formato da alcuni valori o principi e dai diritti che ne rappresentano la traduzione (formale e giuridica). Se si leggono documenti storici e fondanti per l'universo culturale di riferimento, come la Bibbia, il Corano, o antiche mitologie, sembra poter rinvenire elementi comuni, se pur spesso raccontati attraverso parabole e/o declinati come regole di condotta. In primis ciò riguarda ad esempio il concetto di dignità umana, che emerge attraverso la narrazione, trasversale rispetto a diversi orizzonti culturali, del rapporto preferenziale fra il dio e l'uomo ${ }^{16}$. Resta, anche se si riscontra la possibilità di individuare alcuni principi comuni - si pensi anche alla lettura dell'aneddoto di Erodoto prima ricordato -, la difficoltà di accedere ad un concreto piano giuridico. II nucleo comune per potersi concretizzare, cioè per divenire effettivamente universale, paradossalmente, deve particolarizzarsi: l'universalismo vive solo come universalismo contestualizzato o situato. Si postula, dunque, un universalismo che comprende, dal lato teorico, una condivisione di valori o principi (un universalismo essenziale astratto), e, dal lato pratico, un parziale accordo sull'individuazione di alcune posizioni giuridiche (universalismi situati). L'accordo pratico è parziale nel senso che non vi è (necessariamente) perfetta identità nel catalogo dei diritti ma somiglianza: alcuni diritti saranno condivisi, ma potranno avere sfumature differenti, altri diritti saranno più sentiti in certe culture che in altre, ogni cultura presenterà dei propri diritti. In altre parole, il livello di proclamazione dei diritti vive fondamentalmente solo previa contestualizzazione. La formula universalismo situato "copre" poi anche la considerazione che, molto spesso, ed anche nel caso di diritti comuni, i fondamenti e le legittimazioni ad essi sottesi sono differenti, sono parte di storie diverse. È possibile, quindi, distinguere tre livelli: valori o principi, fondamenti o legittimazioni, e catalogo dei diritti. La combinazione prospettata si fonda sulla considerazione che esiste una convergenza (un universalismo) al primo livello, che porta a (o rende possibile) una parziale convergenza

\footnotetext{
${ }^{14}$ Con la precisazione che un ruolo significativo è giocato dalle interpretazioni delle identità culturali (si pensi alla contrapposizione fra tradizionalisti o modernisti nella lettura della shari'a) e dall'approccio (che può essere teso ad evidenziare le convergenze o a mettere in luce le differenze).

${ }^{15}$ La presenza, fra l'altro, di un valore, o di un diritto, orizzontalmente (trasversalmente rispetto a una molteplicità di culture) e/o verticalmente (permanentemente nel corso del tempo), assicura, se pur non con i crismi di una assolutezza fideistica, ma con la ragionevolezza della prospettiva empirica, la stabilità.

${ }^{16}$ Corano, La sura del viaggio notturno (XXVII, 70): «E Noi già molto onorammo i figli d'Adamo e li portammo per la terra e sul mare e demmo loro provvidenza buona, e su molti degli esseri da noi creati preferenza grande» (citazione dalla traduzione curata da A. Bausani, II Corano, Rizzoli, Milano, XXII ed., 2001); Bibbia, Genesi (1, 26-27): «E Dio disse: «Facciamo l'uomo a nostra immagine, a nostra somiglianza, e domini sui pesci del mare e sugli uccelli del cielo, sul bestiame, su tutte le bestie selvatiche e su tutti i rettili che strisciano sulla terra». Dio creò l'uomo a sua immagine...» (citazione dalla versione del testo biblico, ripreso dalla versione italiana della Bibbia curata dalla Conferenza episcopale italiana, contenuta ne La Bibbia di Gerusalemme, Centro editoriale dehoniano, Bologna, 1985); Mitologia makiritare, America: «La donna e l'uomo sognavano che Dio li stava sognando. Dio li sognava mentre cantava e agitava le sue maracas, avvolto in fumo di tabacco, e si sentiva felice e insieme turbato dal dubbio e dal mistero. Gli indios makiritare sanno che, se Dio sogna cibo, fruttifica e dà da mangiare. Se Dio sogna la vita, nasce e dà la nascita» (citazione tratta da E. Galeano, Memoria del fuoco, I - Le origini, Rizzoli, Milano, 1997, p. 7).
} 
(universalismo) nei singoli diritti, anche se il livello intermedio presenta (anche notevoli) differenze. "Universalismo situato", inoltre, appare come un concetto dinamico, in progress, dove la diversità culturale può tradursi in un arricchimento reciproco delle rispettive tradizioni di affermazione concreta dei valori e condurre ad una implementazione del catalogo condiviso dei diritti.

\section{L'«UNIVERSALE IN SENSO LOGICO E AVALUTATIVO»: UNA PROPOSTA DI DEFINIZIONE FORMALE}

Muove, invece, da un approccio che, pur concependo l'universalità come «la principale garanzia» per il rispetto del pluralismo culturale (FERRAJOLI, 2007, p. 58/59) ${ }^{17}$, prescinde dalle culture, Ferrajoli (2007, p. 726), con la sua definizione logica e formale di «diritti fondamentali»: «sono "diritti fondamentali" tutti quei diritti che spettano universalmente a «tutti»»", distinguendosi poi le varie categorie in base alla doppia variabile dell'essere «persone naturali» 0 «cittadini» e del possedere o meno la capacità di agire. II tratto distintivo è «il carattere universale dell'imputazione»; è un universale concepito in «senso logico e avalutativo", per cui l'universalità «è più o meno estesa a seconda dell'estensione delle classi di soggetti» cui i diritti sono riferiti (FERRAJOLI, 2007, p. 726). I diritti che «spettano a tutte le persone naturali semplicemente in quanto tali» sono i diritti umani (FERRAJOLI, 2007, p. 737). È una definizione che lo stesso Ferrajoli (2007, p. 725) qualifica come «formale" e non dice né quali sono i diritti fondamentali e chi ne è titolare (per questo occorre rivolgersi al diritto positivo) né quali devono essere (questione che riguarda la filosofia). L'universalità è elemento portante della struttura del diritto, ma prescinde da qualsiasi riferimento ad una sua fonte, ratio, o modus construendi, ed è "neutra" quanto alla sostanza o all'esistenza fisico-giuridica concreta.

La ricerca di astrattezza e di a-valutazione, al di là dell'indubbio fascino della formula, però, si svigorisce nell'indeterminatezza: l'universalità è indeterminata e, nel tentativo di non perdere in astrattezza e di non assumere qualificazioni implicanti l'adesione ad una prospettiva di valore, assume dei caratteri così vaghi che non consente di identificare nulla a priori come diritto fondamentale e, perdendo ogni carattere prescrittivo, rischia di cadere nella tautologia per cui diritto fondamentale è quello che spetta a "tutti" e ciò che compete a "tutti" è diritto fondamentale. La definizione, in altre parole, pare non definire nulla: è diritto fondamentale ciò che è universale, ma che cos'è universale? Chi stabilisce i termini dell'universalità?

Nella volontà di non uscire da uno spazio avalutativo, la formula proposta si chiude in sé stessa, restando sì aperta a qualsivoglia contenuto, ma senza identificarne o fornire i criteri necessari per ravvisarne alcuno, venendo ad essere un canone di tipo autoreferenziale o relativo (GASTINI, 2001, p. 47) ${ }^{18}$. Emerge la difficoltà - impossibilità? - di passare da una definizione formale alla

\footnotetext{
${ }^{17}$ Ferrajoli, che ravvisa tale garanzia nei diritti di libertà, che garantiscono «l'uguale valore di tutte le differenze personali, a cominciare da quelle culturali», e nei diritti fondamentali quali «leggi del più debole» (ma qui si passa dal piano logico a quello storico dei diritti come consacrati nell'esperienza del costituzionalismo).

${ }^{18}$ In questo senso, Guastini, che rileva il carattere «vuoto» dell'universalità.
} 
sostanza concreta, mantenendo l'astrattezza, senza scendere nella storia ${ }^{19}$. Restando sul piano logico e formale dei diritti fondamentali, si può disquisire delle fallacie del relativismo culturale nella sua critica al paradigma universalistico dei diritti fondamentali (FERRAJOLI, 2007, p 60) ${ }^{20}$, ma, se si ragiona di contenuto, si può prescindere dalle culture?

Si può tentare di rispondere scendendo sulla terraferma concreta del diritto positivo, abbandonando la navigazione fra le teorie dei diritti umani per seguire il sentiero della loro affermazione concreta. La prima questione da affrontare è: i diritti oggi sanciti come universali - nella Dichiarazione universale dei diritti dell'uomo del 1948 e nella sua specificazione nei Patti internazionali sui diritti civili e politici ed economici, sociali e culturali del $1966^{21}$ - sono effettivamente tali?

\section{DIRITTI UNIVERSALI O DIRITTI OCCIDENTALI? UNA COMPARAZIONE FRA LA DICHIARAZIONE UNIVERSALE E LE CARTE REGIONALI DEI DIRITTI UMANI}

La Dichiarazione universale dei diritti dell'uomo del 1948, come è noto, è stata adottata dall'Assemblea Generale delle Nazioni Unite con 48 voti favorevoli, nessun contrario e l'astensione di otto Paesi, fra i quali, peraltro, quelli appartenenti all'(ex) blocco sovietico che avevano partecipato attivamente alla stesura della Dichiarazione e sottoscrivono poi i Patti del 1966.

Nella Dichiarazione universale si incontrano, a prescindere dalla tradizionale matrice giusnaturalista (che scomparirà dai documenti successivi, più "laici"), la prospettiva liberale e la visione socialista, che si coniuga con un certo umanesimo cattolico o, comunque, con le istanze sociali presenti anche nei Paesi occidentali (si pensi all'umanesimo integrale di Maritain (1953, p. 129) o alla rooseveltiana libertà dal bisogno ${ }^{22}$ ). L'idea liberale della completa autonomia dell'individuo rispetto allo Stato si mitiga: l'individuo deve essere libero dallo Stato, ma anche dai bisogni che ostacolano il libero sviluppo della sua persona, e deve potersi estrinsecare non solo come monade isolata, ma anche come membro di "società". L'individualismo negativo dell'epoca liberale cede il passo alla concezione di eguaglianza sostanziale e di valorizzazione del pluralismo dello stato sociale: ai diritti individuali di libertà negativa si affiancano diritti collettivi e diritti economici e sociali. II titolare dei diritti è una persona umana situata in un determinato contesto economico-sociale e (almeno potenzialmente) inserita in una rete di formazioni sociali: le "pretese" di questa persona umana sono i diritti proclamati universali. La prospettiva è quella dei diritti (solo un cenno generico è fatto ai doveri) ${ }^{23}$ e il dominus dei diritti è l'individuo; le associazioni e le comunità intermedie paiono tutelate non tanto

\footnotetext{
${ }^{19}$ Sulle difficoltà della teoria di Ferrajoli di mantenersi unicamente su un piano logico-formale, cfr. D. Zolo, Libertà, proprietà ed eguaglianza nella teoria dei "diritti fondamentali", in L. Ferrajoli, Diritti fondamentali, cit., pp. 49 ss.

${ }^{20}$ Così L. Ferrajoli, che discorre di tre fallacie, una di tipo logico e metaetico, una di tipo giuridico ed una eticapolitica.

${ }^{21}$ Tali documenti si possono considerare la "costituzione mondiale dei diritti umani"; i patti e le dichiarazioni successive, da un lato, specificheranno, in relazione all'oggetto e ai titolari, posizioni giuridiche già contenute nel catalogo "1948-1966", dall'altro, aggiorneranno il catalogo, tenendo conto degli sviluppi tecnologici e delle nuove conquiste (come il diritto all'acqua).

${ }^{22}$ Cfr. il messaggio del Presidente Roosevelt al Congresso del 6 gennaio 1941, nonché la Dichiarazione comune adottata il 14 agosto 1941 da Roosevelt e Churchill (c.d. Carta Atlantica).

${ }^{23}$ "Ogni individuo ha dei doveri verso la comunità» (Dichiarazione universale, art. 29, par. 1).
} 
in quanto soggetti (autonomi) a fianco dell'individuo, ma piuttosto in quanto "funzionali" rispetto ad una piena espressione dell'individuo.

La Dichiarazione universale è, in altre parole, sostanzialmente espressione di una concezione imperniata sulla singola persona, anche se non si tratta più dello sfrenato individualismo possessivo borghese, ma di un individualismo "sociale", mite ed aperto, solidale.

I Patti del 1966 non muteranno l'impostazione, anche se registreranno l'introduzione del diritto di autoderminazione in capo ai «popoli» ${ }^{24}$, in recezione di istanze provenienti dai Paesi del c.d. Terzo Mondo ${ }^{25}$.

Questi diritti rappresentano veramente un «ideale comune da raggiungersi da tutti i popoli e da tutte le Nazioni», come è proclamato nel Preambolo della Dichiarazione universale? Possono costituire un quid universale anche per culture dove centrale è la prospettiva comunitarista e l'ottica del dovere?

Si pensi al concetto indù di dharma, che, all'interno di un ordine cosmico fondato su un equilibrio armonico di tensioni, evoca modelli di comportamento orientati secondo l'idea del dovere, in una commistione fra diritto ed etica. É il contesto per cui «tutti i diritti dell'uomo degni di essere meritati e conservati sono quelli dati dal dovere compiuto» (Gandhi, 1948). O, ancora, sempre per un'impostazione nel senso del dovere, si ricordi il sistema nipponico dei giri, regole di condotta basate sul ruolo sociale ricoperto, inserite in un orizzonte dove predominano il wa (armonia) o il mura (comunità solidale), con una propensione per la conciliazione piuttosto che per la rivendicazione di prerogative $^{26}$. L'approccio deontico si sposa con il riconoscimento del ruolo centrale della comunità, come soggetto di diritti e, se non prioritaria, almeno sullo stesso piano degli individui, sia essa la umma del diritto islamico o il clan dei contesti tribali tradizionali. È il concetto africano di ubuntu ${ }^{27}$, per cui si è persona solo nel rapporto con altre persone, all'interno di un gruppo sociale, che segna la distanza da un taglio individualistico-atomistico.

La prospettiva "comunitarista-deontica" è compatibile con diritti universali strutturati essenzialmente come pretese individuali? Può trovare estrinsecazione come contestualizzazione locale, accanto a diritti sanciti a livello globale? Una risposta può essere cercata nelle carte che, a

\footnotetext{
${ }^{24}$ Patto sui diritti civili e politici, parte I, art. 1, par. 1 e 2; nonché, ugualmente, Patto sui diritti economici, sociali e culturali, parte I, art. 1, parr. 1 e 2.

${ }^{25}$ Come dato negativo per il cammino dell'universalità può invece essere letto lo sdoppiamento dei Patti, che segnala una frattura nella rappresentazione dei diritti dell'uomo, separando i valori di libertà (negativa) e di giustizia sociale e solidarietà.

${ }^{26}$ Propensione, quella per una risoluzione armonica e conciliativa delle controversie, che si trova anche in molti sistemi di giustizia tradizionale africana (come le corti Gacaca in Ruanda).

${ }^{27}$ Sotto la voce Ubuntu, nel White Paper for Social Welfare (cap. 2, par. 24), adottato nel 1997 dal governo sudafricano, si legge: «The principle of caring for each other's well-being will be promoted, and a spirit of mutual support fostered. Each individual's humanity is ideally expressed through his or her relationship with others and theirs in turn through a recognition of the individual's humanity. Ubuntu means that people are people through other people. It also acknowledges both the rights and the responsibilities of every citizen in promoting individual and societal well-being» (http://www.ilo.org/public/english/region/asro/bangkok/ability/download/zaf_wp.htm).
} 
livello continentale, costituiscono il catalogo dei diritti umani ${ }^{28}$. Fra le carte più interessanti sotto questo profilo vi è indubbiamente quella africana, la Carta di Banjul dei diritti dell'uomo e dei popoli, adottata in ambito OUA, nel 1981.

In tale Carta vi è, innanzitutto, una forte affermazione dell'identità africana, in specie in contrapposizione al recente passato coloniale e all'affermarsi di pratiche di neo-colonialismo, e, in secondo luogo, un riconoscimento della Dichiarazione dei diritti dell'uomo del 1948, che è tenuta nella «dovuta considerazione», $\mathrm{ma}$ in un contesto nel quale sono «le virtù della... tradizione storica $\mathrm{e} i$ valori della civiltà africana» che ispirano e caratterizzano le «riflessioni sul concetto di "diritti dell'uomo e dei popoli"” (dal Preambolo). Non si rifiutano, dunque, i diritti della persona umana nati in Occidente e considerati universali, ma si sostiene, da un lato, la loro effettiva universalità, cioè il fatto che essi non sono un prodotto solo della cultura occidentale, ma affondano le loro radici anche nelle tradizioni africane, e, dall'altro, si indicano alcune specificità della situazione africana, sì da situare l'universalismo e da prospettare una via "africana" ai diritti universali. In altre parole, non si nega la possibilità di raggiungere un accordo pratico sui diritti universali e, magari, anche una convergenza intorno ad alcuni valori, ma si rivendica una propria legittimazione o fondamento dei diritti (le tradizioni africane) e una propria specificazione e integrazione del catalogo dei diritti. Nella Carta, quindi, accanto alla proclamazione dei classici diritti di libertà, politici e sociali, vi è il riconoscimento del diritto allo sviluppo, della centralità della comunità, dei diritti dei popoli, dell'importanza dei doveri.

Un discorso diverso riguarda invece il continente americano, con la Convenzione americana sui diritti dell'uomo, stipulata a San José de Costarica, nel 1969, con l'intenzione «di consolidare in questo emisfero, nel quadro di istituzioni democratiche, un sistema di libertà personali e di giustizia sociale basato sul rispetto dei diritti essenziali dell'uomo" (Preambolo). La prospettiva prevalente è di tipo soggettivista e strutturata sulla falsariga della rivendicazione di una pretesa ${ }^{29}$ : vi è dunque omogeneità con l'immediato retroterra e la tipologia delle posizioni giuridiche soggettive dei documenti internazionali del 1948 e 1966. Rimane la considerazione - più di un dubbio, (quasi) una certezza che l'omogeneità o la vicinanza oggi riscontrabili siano il frutto di una universalizzazione forzata compiuta nel passato: la condivisione è fra la cultura dei colonizzatori e la cultura dei loro conterranei dei Paesi di provenienza, con l'esclusione (o l'eliminazione) delle culture dei popoli autoctoni, al più tutelate in quanto espressione di una minoranza. Forse, se si confronta quella cultura che si riconosce, ad esempio, nel Popol Vuh, il libro sacro dei Maya ${ }^{30}$, con quella maggioritaria degli ex colonizzatori si possono trovare valori in comune (la dignità umana), ma anche impostazioni differenti (di nuovo l'idea del dovere più che quella del diritto, la centralità della comunità): solo che è

\footnotetext{
${ }^{28}$ Non si prendono in esame in questa sede le carte dei diritti adottate in ambito europeo dato che il loro sostrato, ed il catalogo dei diritti, appartengono allo stesso universo cultural-giuridico dei diritti umani internazionali.

${ }^{29}$ I diritti economico-sociali, tuttavia, sono solo oggetto di un generico impegno (Cap. III, art. 26); saranno poi meglio specificati nel Protocollo addizionale sui diritti economici, sociali e culturali, approvato a San Salvador nel 1988.

${ }^{30}$ "Figli: ovunque siate non venite meno ai doveri che vi insegnò Ixpiyacoc, perché sono doveri che vengono dalla tradizione dei vostri padri» (la citazione del Popol Vuh è tratta da E. Burgos (cur.), Mi chiamo Rigoberta Menchù, Firenze, Giunti, 1987, al quale si rimanda per una prima indicazione sui contenuti della cultura maya e, in particolare, quiché).
} 
considerata al più come una cultura da tutelare dall'estinzione, non come capace di indicare una via americana (o, meglio, maya, inca, urone, etc.) ai diritti. Oggi, peraltro, l'esistenza di "altre" culture inizia ad emergere a livello costituzionale: le più recenti costituzioni latinoamericane, oltre a dedicare un'attenzione maggiore alla tutela delle diversità culturali, recepiscono alcune istanze comunitariste 0 valori culturali tradizionali ${ }^{31}$.

Quanto al continente asiatico, si può qui limitarsi ad osservare come, nonostante il vivace dibattito intorno agli Asian values, manchi una vero e proprio accordo continentale sui diritti. Ciò che ci si avvicina di più è la Carta asiatica dei diritti umani, adottata nel 1998 dalla Commissione asiatica dei diritti dell'uomo, un organismo non governativo indipendente creato da un gruppo di giuristi asiatici, senza, dunque, alcuna efficacia giuridica. È interessante, nella Carta, la risposta data alla querelle universalità-identità culturali. Si ritiene possibile o, meglio, necessario, contemperare universalità e tradizioni culturali e si afferma che occorre «muovere dalle formulazioni astratte e procedere verso la concretizzazione dei diritti fondamentali nel contesto asiatico... » (par. 2.3): un universalismo situato?

Merita infine un breve approfondimento la Dichiarazione dei diritti dell'uomo nell'Islam, adottata al Cairo nel 1990, nell'ambito dell'Organizzazione della Conferenza islamica, quale emblema delle carte dei diritti fortemente connotate dall'orizzonte religioso di riferimento.

Come nella Carta di Banjul, il documento si apre con una rivendicazione del valore della cultura di appartenenza e con una riconduzione dei diritti a tale cultura: «nella certezza che i diritti fondamentali e le libertà universali nell'Islam facciano parte della religione islamica» (Preambolo). I diritti, però, in tal caso sono «disposizioni divine», da osservare e proteggere attraverso un «atto di adorazione» (Preambolo), sono cioè inscritti in una visione religiosa, di tipo monoteista e (almeno) potenzialmente totalizzante: come influisce tale dato sull'universalità dei diritti?

La shari'a è un elemento onnipresente nella Dichiarazione del Cairo, dal Preambolo alle disposizioni finali di chiusura con le quali si subordinano «tutti i diritti e le libertà enunciati... alle disposizioni contenute nella Legge islamica» (art. 24), passando per il riconoscimento dei singoli. Scorrendo il testo della Dichiarazione, infatti, si incontrano i classici diritti di libertà, politici e sociali, ma con la clausola che il loro esercizio avvenga «conformemente alla Legge islamica». Ora, se è evidente che il sottile confine fra contestualizzazione dei diritti della persona umana e creazione di un "altro" sistema dei diritti (o, ancor meglio, di affermazione di valori), è varcato con maggior facilità nell'ipotesi di costruzioni culturali onnicomprensive, d'altra parte, è anche vero che, per la maggior parte, i fondamenti si presentano come verità assolute ed incontestabili, come i migliori o gli unici configurabili, ma ciò non esclude la possibilità di individuare un quid comune o di raggiungere un accordo su un catalogo dei diritti. In linea teorica è possibile, come si è detto, ipotizzare l'esistenza di diritti condivisi e, contemporaneamente, di strumenti di legittimazione differenti: molto dipende, in sostanza, dal contenuto della Legge islamica (dalla distinzione, ad esempio, fra valori e prescrizioni "storiche") e dalla sua interpretazione (tradizionalista o modernista).

\footnotetext{
${ }^{31}$ Per tutte si citano la Constitución de la República del Ecuador del 2008, Tit. II, Cap. II, con i derechos del buon vivir, o Cap. IV, con i derechos de las comunidades, pueblos y nacionalidades; e la Nueva Constitución política del Estado della Bolivia del 2007, spec. Tit. II, Cap. IV, Derechos de las naciones y pueblos indígena originario campesinos, e Cap. VI, Educación, interculturalidad y derechos culturales.
} 
L'excursus fra le carte continentali mostra che, se si segue un'interpretazione rigidamente ancorata al diritto positivo vigente, per la quale i diritti universali sono solo i diritti scritti nella Dichiarazione universale del 1948 (e nei Patti del 1966), come interpretati alla luce del loro specifico retroterra socio-culturale, si può dubitare della loro esistenza. Si potrebbe quindi configurare un doppio scenario: da un lato, una universalità tramutata in imposizione, in una forma di imperialismo (culturale e giuridico); dall'altro, la conclusione nel senso dell'impossibilità di configurare diritti validi per ciascuna persona umana.

La soluzione, tuttavia, potrebbe essere diversa se si ipotizza una lettura "debole" dei diritti del 1948 o del 1966, visti come suscettibili di integrazioni e adattamenti, alla luce dei differenti contesti, e preventivamente sfrondati degli aspetti più direttamente legati alla matrice liberale. In altri termini, occorrerebbe operare una de-contestualizzazione del diritto internazionale dei diritti umani vigente, sino ad incontrare il loro nocciolo universale, da declinare quindi nei vari contesti.

Inoltre, perché i diritti possano effettivamente vivere ed essere vissuti come universali è necessario rimuoverne le ambiguità ed evitarne le strumentalizzazioni.

\section{UNIVERSALITÀ ALLA PROVA: LE AMBIGUITÀ E I RISCHI DI STRUMENTALIZZAZIONE}

Le ambiguità dell'universalità emergono nitidamente con l'immigrazione dalle terre del Sud del mondo verso l'Occidente. I migranti portano in superficie, proprio nella culla dei diritti universali, le crepe profonde che si creano nella collisione fra universalità e sovranità dello stato e, in particolare, fra universalità e cittadinanza, nonché fra universalità e identità culturali.

Emerge che, quando, nello scenario internazionale, si scontrano la soggettività della persona, con i suoi diritti universali, e quella degli Stati, con la loro sovranità, a soccombere molto spesso è la prima. Un esempio emblematico: il riconoscimento monco della libertà di circolazione transnazionale. La Dichiarazione universale e i Patti del 1966 sanciscono il diritto di ogni individuo di «lasciare qualsiasi paese, incluso il proprio» ${ }^{32}$, senza la previsione, con una evidente illogicità, del corrispondente diritto di entrare in un altro paese, mantenendo immutata l'asimmetria di fondo dello ius migrandi di Vitoria. Gli Stati mantengono il controllo sui propri confini, il potere di decidere, al di là dei propri cittadini ${ }^{33}$, chi e a quali condizioni può accedere e permanere nel proprio territorio: le "semplici" persone umane, paradossalmente, hanno il "diritto" di vivere nelle acque internazionali, in perpetua navigazione, o nella terra di nessuno al confine tra due Stati, tra le rispettive barriere di filo spinato.

Trova conferme l'osservazione della Arendt (1997, p. 145): «la perdita dei diritti nazionali ha portato con sé in tutti i casi la perdita dei diritti umani... La concezione dei diritti umani è naufragata nel momento in cui sono comparsi individui che avevano perso tutte le altre qualità e relazioni specifiche, tranne la loro qualità umana»?

\footnotetext{
${ }^{32}$ Cfr. Dichiarazione universale del 1948, art. 13, par. 1; Patto internazionale sui diritti civili e politici del 1966, art. 12, par. 1.

${ }^{33}$ Laddove sia riconosciuto il diritto di asilo, anche i richiedenti asilo possono vantare un diritto di ingresso nel territorio di uno Stato, quantomeno per il tempo necessario all'esame della domanda.
} 
I diritti sono universali, ma, in alcuni casi, per esserne titolari occorre essere anche cittadini. Si pensi ai diritti politici: i documenti internazionali sui diritti dell'uomo, generalmente, o riconoscono direttamente i diritti di partecipazione al governo in capo al cittadino o li attribuiscono ad «ogni individuo», ma riferendosi al governo del «proprio paese» ${ }^{34}$, sottintendendo con questa ultima espressione, tradizionalmente, "stato di cui si è cittadini".

Il legame commutativo fra diritti politici e cittadinanza (gode dei diritti politici chi è cittadino e l'esercizio dei diritti politici contraddistingue lo status del cittadino) rafforza poi l'esclusione dalla titolarità dei non cittadini, anche se regolarmente e stabilmente residenti; le aperture, con l'estensione del diritto di voto allo straniero titolare di permesso di soggiorno da un certo numero di anni, sono timide: previste solo in via legislativa e limitate al livello delle elezioni locali.

In altre ipotesi, la cittadinanza costituisce un criterio per discriminare nel godimento dei diritti, come avviene soprattutto in relazione ai diritti sociali. La prassi normativa degli Stati è nel senso di graduare, perlomeno per alcuni diritti sociali (quale la salute), l'esercizio in rapporto allo status del suo potenziale titolare. II diritto sociale viene scisso: il nucleo minimo, che tutela negli aspetti più essenziali la vita della persona umana, e il contenuto che, si potrebbe dire, garantisce un livello di vita medio rispetto a quello dei cittadini. II nucleo minimo è riconosciuto a tutti: cittadini, stranieri regolari e stabilmente presenti, stranieri irregolari o presenti solo occasionalmente; la tutela piena, invece, è riconosciuta, in condizione di parità con i cittadini, solo agli stranieri regolari o appartenenti a categorie cui è accordata una speciale protezione, quali i minori e le donne incinte.

Vi sono, dunque, di fatto, diritti universali della persona umana, ovunque si trovi e al di là di ogni sua ulteriore caratterizzazione, diritti universali della persona umana riservati ai cittadini, diritti universali della persona umana il cui godimento è subordinato al possesso di alcuni titoli e dipende, nel grado di tutela, dal titolo posseduto.

Lo status persona umana si liquefa nella distinzione fra cittadino, straniero, richiedente asilo, straniero regolarmente soggiornante e straniero irregolarmente presente sul territorio. L'universalità rischia di degradare - o, forse meglio, è degradata - ad un mero livello retorico.

A ciò può aggiungersi la considerazione che, mancando una effettiva universalità nella tutela dei diritti a livello globale ${ }^{35}$, il loro concreto esercizio dipende dalla residenza - e dalla cittadinanza - in un singolo Paese: si godrà, dunque, dei diritti universali diversamente a seconda della tutela riconosciuta in ciascuno Stato. L'universalità si frammenta nelle sovranità statali.

Con una aggravante: la residenza non è oggetto di una libera scelta, ma dipende dai criteri fissati discrezionalmente dai vari Stati (le norme sulla cittadinanza e quelle sull'ingresso e il soggiorno degli stranieri); lo straniero, in particolare, avrà nello Stato "ospite" dei diritti universali ed inviolabili ma allo stesso tempo "temporanei e sub condicione", fintantoché perdura il beneplacito al suo soggiorno da parte dello Stato stesso.

L'universalità, inoltre, si anticipava, viene revocata in dubbio anche con l'ingresso nella cultura occidentale/universale di tradizioni culturali differenti, che più facilmente innestano il conflitto fra singoli

\footnotetext{
${ }^{34}$ Cfr. Dichiarazione universale del 1948, art. 21; Patto internazionale sui diritti civili e politici del 1966, art. 25.

${ }^{35}$ Si pensi in primis alla mancanza di un tribunale mondiale dei diritti umani, in grado di garantire una tutela giurisdizionale ai diritti proclamati come universali; mancanza che non è sanata da organi come la Corte penale internazionale, dati i suoi limiti di competenza (le gross violations) ed operatività.
} 
diritti universali e prescrizioni culturali tutelate dal riferimento al diritto all'identità culturale. L'universalità, se troppo "accomodante", rischia di dissolversi per l'invadenza delle prescrizioni che il diritto all'identità culturale veicola, mentre, se troppo "rigida", rischia di risolversi in una imposizione e tramutarsi in imperialismo.

In questo senso è da valutare anche la dipendenza dell'universalità dal significato politico che i diritti assumono o con il quale vengono percepiti, dall'“uso" politico che ne viene fatto. Se i diritti vengono utilizzati strumentalmente, quali arma ideologica o quale ideologia al seguito di operazioni belliche o di controllo economico, perdono ogni aura di universalità. Si pensi, emblematicamente, alla cosiddetta ingerenza umanitaria, ossia al diritto per i singoli Stati di intervenire con la forza armata ovunque i diritti umani siano violati. Quello che a prima vista potrebbe apparire un nuovo cammino verso una tutela effettiva ed universale, si risolve in realtà nel ritorno della vecchia "legge del più forte". Da un lato, infatti, in questi casi i diritti vengono presentati attraverso una imposizione (una nonlibertà) e spesso le armate che li "accompagnano" causano un aumento delle loro violazioni; dall'altro, essi (spesso) non sono che il vessillo dietro cui si mascherano interessi economico-finanziari e politici.

\section{OSSERVAZIONI CONCLUSIVE}

I diritti mostrano il loro doppio volto: sono «veleno e cura», in quanto «possono essere, sono stati, ancora sono, strumento di un imperialismo culturale che apre la strada all'imperialismo economico e sociale attraverso la distruzione delle culture che sono incompatibili con lo sviluppo economico, sociale e politico di cui essi sono presupposto», ma, nello stesso tempo, sono «anche argine e difesa» contro l'imperialismo stesso (PITCH, 1995, p. 95) ${ }^{36}$.

L'assunzione di una prospettiva di costruzione e interpretazione dei diritti trans-culturale sulla falsariga di concetti come l'universalismo situato, coniugata con una corretta percezione della duplicità e ambiguità dei diritti ${ }^{37}$, può allontanare lo spettro del loro volto "cattivo" e rappresentare il contesto per lo sviluppo di diritti effettivamente universali. Ciò accanto ad una tutela effettiva, e democratica, a livello globale; come ricordava Bobbio (1992, p. 16), «il problema di fondo relativo ai diritti dell'uomo è oggi non tanto quello di giustificarli, quanto quello di proteggerli».

Oggi peraltro la globalizzazione dei diritti pare recessiva, o, si potrebbe dire, vi è una recessione globale nei diritti. In primo luogo, la strada per una giustizia penale internazionale pare ancora lunga, come mostrano le difficoltà incontrate nella costituzione della Corte penale internazionale, pur limitata nella sua competenza. In secondo luogo, accanto alla timida nascita di

\footnotetext{
${ }^{36}$ similmente, cfr. anche S. Latouche, L'occidentalisation du monde. Essai sur la signification, la portée et les limites de l'uniformisation planétaire, Paris, 1989, trad. it. L'occidentalizzazione del mondo, Bollati Boringhieri, Torino, 1992, p. 142: l'Occidente ha utilizzato i diritti in funzione imperialista, ma «parallelamente alla deculturazione del pianeta e all'imperialismo sotto tutte le sue forme,... ha prodotto ed elaborato il sogno di una città emancipata dove tutti gli uomini avrebbero il loro posto e della quale ciascuno sarebbe un libero cittadino»; in tema, cfr. anche S. Žižek, Contro i diritti umani, il Saggiatore, Milano, 2005.

37 Il senso dell'esposizione delle ambiguità dei diritti è quello espresso da E. Balibar, Le frontiere della democrazia, manifestolibri, Roma, 1993: «penso che l'esplicitazione delle aporie della politica dei diritti dell'uomo rappresenti già una delle condizioni di esistenza di essa. Per questo parlo dell'universalità e dei suoi paradossi...» (p. 15, nonché, più ampiamente, pp. 183 ss.).
} 
nuovi diritti, come il diritto all'acqua ${ }^{38}$, si assiste ad una inversione di rotta nella garanzia di altri. Da un lato, i classici diritti di libertà, in primis la libertà personale e quella di manifestazione del pensiero, hanno subito limitazioni in nome della sicurezza da parte delle discipline antiterrorismo adottate in molti Paesi dopo l'11 settembre 2001; dall'altro lato, i diritti sociali e del lavoro vengono smantellati in nome delle esigenze del pareggio di bilancio e degli effetti della crisi.

Forse - si può osservare in conclusione - l'universalità non esiste solo nel canto delle sirene di chi postula l'esistenza o immagina la costruzione di diritti della persona umana, ma la sua voce avrebbe bisogno di una maggior polifonia, di risuonare anche nelle aule di un tribunale, e - non ultimo - di trovar sempre cantori disponibili a non farsi ridurre al silenzio, perché i diritti non sono sottratti al «flusso della storia» ${ }^{39}$, con i suoi corsi e ricorsi.

\section{BIBLIOGRAFIA}

ARENDT, Hannah. Le origini del totalitarismo (1967), Edizioni di Comunità, Milano, 1997.

AZZARITI, Gaetano. Diritto e conflitti. Lezioni di diritto costituzionale, Laterza, Roma-Bari, 2010.

BALIBAR, Etienne Le frontiere della democrazia, manifestolibri, Roma, 1993.

BAUSANI, Alessandro. II Corano, Rizzoli, Milano, XXII ed., 2001

BERTI, Enrico., Per una fondazione filosofica dei diritti umani, in Id., Le vie della ragione, il Mulino, Bologna, 1987, p. 290.

BOBBIO, Norberto. Locke e il diritto naturale, Giappichelli, Torino, 1963.

BOBBIO, Norberto. Sul fondamento dei diritti dell'uomo, in Id., L'età dei diritti, Einaudi, Torino, 1992.

BURKE, Edmund. Riflessioni sulla Rivoluzione francese e sulle deliberazioni di alcune società di Londra ad essa relative: in una lettera destinata ad un gentiluomo parigino, 1790, in Scritti politici, UTET, Torino, 1963.

D'AGOSTINO, Francesco. Pluralità delle culture e universalità dei diritti, in Id. (a cura di), Pluralità delle culture e universalità dei diritti, Giappichelli, Torino, 1996.

DAHL, Robert Alan On Democracy, 1998, trad. it. Sulla democrazia, Laterza, Roma-Bari, 2000.

FERRAJOLI, Luigi. Diritto e ragione. Teoria del garantismo penale, Laterza, Roma-Bari, 1989.

FERRAJOLI, Luigi. Principia iuris. Teoria del diritto e della democrazia, vol. 2. Teoria della democrazia, Laterza, Roma-Bari, 2007.

GALEANO, Eduardo. Memoria del fuoco, I - Le origini, Rizzoli, Milano, 1997.

GEERTZ, Clifford. Mondo globale, mondi locali. Cultura e politica alla fine del ventesimo secolo, il Mulino, Bologna, 1999.

GROZIO, Ugo. I prolegomeni al De jure belli ac pacis, trad. it. di S. Catalano, Palumbo, Palermo, 1948.

38 Vedi United Nations, General Assembly, risoluzione del 28 luglio 2010 (GA/10967, www.un.org/News/Press/docs/2010/ga10967.doc.htm).

${ }^{39}$ N. Bobbio, Presente e avvenire dei diritti dell'uomo, in Id,, L'età dei diritti, cit., p. 26. 
GUASTINI, Riccardo. Tre problemi di definizione, in L. Ferrajoli, Diritti fondamentali. Un dibattito teorico, a cura di E. Vitale, Laterza, Roma-Bari, 2001.

HUNTINGTON, Samuel P. The Clash of Civilizations and the Remaking of World Order (1996), trad. it. Lo scontro delle civiltà e il nuovo ordine mondiale, Garzanti, Milano, 2000.

IGNATIEFF, Michel. Una ragionevole apologia dei diritti umani, Feltrinelli, Milano 2003.

JULLIEN, François. De l'universel, de l'uniforme, du commun et du dialogue entre les cultures, Paris, 2008, trad. it. L'universale e il comune. II dialogo tra culture, Laterza, Roma-Bari, 2010, pp. 124-125.

LATOUCHE, Serge, L'occidentalisation du monde. Essai sur la signification, la portée et les limites de l'uniformisation planétaire, Paris, 1989, trad. it. L'occidentalizzazione del mondo, Bollati Boringhieri, Torino, 1992.

LATOUCHE, Serge. L'occidentalizzazione del mondo quindici anni dopo, in Dem. e dir., 4/2004.

LOCKE, John. Epistola de Tolerantia, 1689, trad. it. Lettera sulla tolleranza, Laterza, Roma-Bari, 1998.

MARITAIN, Jacques Les droits de l'homme et la loi naturelle, 1942, trad. it. I diritti dell'uomo e la legge naturale, Milano, Edizioni di Comunità, 1953.

NUSSBAUM, Martha Craven. Giustizia sociale e dignità umana. Da individui a persone, il Mulino, Bologna, 2002.

PITCH, Tammar. L'antropologia dei diritti umani, in A. Giasanti, G. Maggioni, I diritti nascosti. Approccio antropologico e prospettiva sociologica, Raffaello Cortina Editore, Milano, 1995.

RAWLS, John. A theory of justice (1971), trad. it. Una teoria della giustizia, Feltrinelli, Milano, 1997.

RAWLS, John. La legge dei popoli, in S. Shute, S. Hurley (a cura di), I diritti umani. Oxford Amnesty Lectures 1993, Garzanti, Milano, 1994.

ROULAND, Norbert. I fondamenti antropologici dei diritti dell'uomo, in Rivista internazionale di filosofia del diritto, 1998.

SEN, Amartya. La democrazia degli altri. Perché la libertà non è un'invenzione dell'Occidente, Mondadori, Milano, 2004.

VECA, Salvatore. Dell'incertezza. Tre meditazioni filosofiche, Feltrinelli, Milano, 1997.

VECA, Salvatore. I diritti umani e la priorità del male, in M. Ignatieff, Human rights as politics and idolatry (2001), trad. it. Una ragionevole apologia dei diritti umani, Feltrinelli, Milano, 2003.

WALZER, Michael. Due specie di universalismo, in MicroMega, 1991.

ŽlŽEK, Slavoj. Contro i diritti umani, il Saggiatore, Milano, 2005.

ZOLO, Danilo Libertà, proprietà ed eguaglianza nella teoria dei "diritti fondamentali", in L. Ferrajoli, Diritti fondamentali. Un dibattito teorico, a cura di E. Vitale, Laterza, Roma-Bari, 2001.

\section{COMO CITAR ESSE DOCUMENTO}

ALGOSTINO, Alessandra. I Diritti Umani e la sfida dell'Universalità. Revista do Direito, Santa Cruz do Sul, v. 2, n. 49, maio 2016. ISSN 1982-9957. Disponível em: $<$ https://online.unisc.br/seer/index.php/direito/article/view/7893>. Acesso em: doi:http://dx.doi.org/10.17058/rdunisc.v2i49.7893. 